\title{
Cavity Soliton Molecules and All-Optical Push-Broom Effect
}

\author{
Baldeep Kaur and Soumendu Jana (1)
}

\begin{abstract}
We present an improved model for cavity soliton generation by coupling frequency-selective feedback to a verticalcavity surface emitting laser with a saturable absorber. The frequency-selective feedback is found to lower down the threshold pump power for cavity soliton generation and eventually to broaden the stability regime. A new dynamics of cavity solitons is revealed in form of "all-optical push-broom effect." Also, stable bound states of cavity solitons that resemble linear diatomic and polyatomic molecules are predicted with their stability regions marked. The findings can be exploited for experimental realization of a soliton force microscope and all-optical memory devices.
\end{abstract}

Index Terms-All-optical push-broom, bound states, cavity solitons, complex Ginzburg-Landau equation.

\section{INTRODUCTION}

$\mathbf{C}$ AVITY soliton (CS) attracted significant research interest due to its application as future "bit" of information and reconfigurable pixels and all-optical memory [1]-[6]. CSs are bright spots on dark background or dark spots on the bright background. They can be generated in a broad area device like vertical-cavity surface emitting laser (VCSEL) with the help of a holding beam that compensate the loss of the dissipative system [1]. The need of holding beam and the corresponding complications (e.g., thermal effects) can be removed either by coupling a frequency-selective feedback (FSF) with the VCSEL [7]-[10] or using a saturable absorber (SA) in a VCSEL [1], [11]-[13]. Both of these configurations lead to a cavity soliton laser (CSL) [1], [7]-[13]. VCSEL-FSF model uses an external Bragg reflector (to provide feedback) coupled with a grating for frequency selection [8]. Although otherwise successful, a CS system needs a bistability between the lasing and non-lasing state, which a standard laser usually lacks. VCSEL-SA model can provide such bistability. But, in turn SA produces undesired loss. Available literature reports the theoretical study and experimental realization of CS and CSL in VCSEL coupled either with SA [11]-[13] or FSF [8], [14]-[18], but a combination of them has not paid a proper attention in spite their potential to compensate each other's drawbacks. Thus, we propose a combined model where VCSEL with SA layer is coupled with FSF. This VCSEL-SA-FSF model aims to reap the benefit of the symbiotic behavior of FSF and SA; while SA will provide bistability, FSF will provide the gain to the system. Moreover, such integrated configuration may lowers down the required pump power. Nevertheless, this improved system (VCSEL-SA-FSF) may lead to intriguing novel CS dynamics, which is not a little less important. Particularly, the CS dynamics and interaction are of much interest due to their potential implications in future all-optical devices. A CS can be excited at any desired point of the transverse plane of the cavity. The stationary CS can be set in motion, in principle, by breaking translational symmetry due to the spatial modulation on any system parameter. Gradients in the cavity parameters, such as intensity [19], phase [19]-[21], detuning [22], amplitude [19], [21], [22], thermal [21], [22], inhomogeneous cavity resonance [5], [21], cavity length [5] and field gradients [23] play a major role in defining the dynamics of the CSs. The uniform variation of detuning parameter in the semiconductor resonator (i.e., VCSEL) also sets a gradient [5]. Any gradient or inhomogeneity in the media leads to the movement of CS [24]. Gradients in the cavity can be introduced through injection beam [23], time delay, feedback field strength [15], [16], feedback field phase [14] or by generating gradients in the cavity background [5]. A moving CS can come to rest when it attains an equilibrium state or the corresponding gradient vanishes [23]. CS dynamics can be alternatively envisaged through a bifurcation of the system, which can be done by exploiting variation of a suitable cavity parameter. The spontaneous and gradient driven movements of CSs are studied in VCSEL with periodic phase modulation of injected beam, as well as detuning [22]. CS dynamics and interactions in broad-area semiconductor laser (particularly VCSEL) coupled with FSF is reported in [25]. Theoretical prediction is made for the existence and stability of oscillating, oscillating-rotating and traveling twin CS pair [13]. The interaction of attractive and repulsive forces between two CSs binds them to a particle-like state. This bound state of CS is referred as CS molecule. CS molecules have fixed relative positions, but a continuously evolving relative phase. Depending on the cavity parameters CS molecules can rotate, oscillate and travel [13]. Various soliton molecules are formed in 
semiconductor micro-cavity, e.g., elliptically polarized polariton soliton molecules [26]. Soliton molecules can be originated in fiber laser cavity as well [27], [28]. Both an anomalous and an all-normal dispersion fiber cavity [27] can support soliton molecules. A pair of group-velocity-locked vector solitons also exhibit molecule formation [28]. Both the relative phase and separation between CS change during interactions [29]. Interactions of CSs in higher order nonlinear medium, like materials exhibiting saturable nonlinearities or higher order nonlinearities, other than Kerr nonlinearity, presents intriguing dynamics such as fission, fusion and annihilation [30]. Two mutually inphase coherent CSs always experience the attraction, whereas, out-of-phase CSs always lead to the repulsion. However, the intermediate phase difference results in the interactions influenced by the energy exchange. Intensity in the overlapping region increases (decreases) for the in-phase (out-of-phase) CSs. This results in the local increase (decrease) of refractive index in the material, and eventual attraction (repulsion) between the CSs. However, in homogeneous materials, generally the phase difference of the interacting solitons explicitly influence the trajectories of motion [31]. The afore mentioned CS dynamics can be used for realization of actual device only if the dynamics is controllable as well as predictable. Our proposed model looks forward to provide such desired control on CS dynamics by means of tuning the FSF. An intriguing CS dynamics can be envisaged in form of controlling a CS by another. Such controllable CS works as an all-optical "push-broom". Although few reports [32]-[34] used the term "push-broom", but those were merely compression of the signal pulse not an actual movement of the soliton. We also aim to explore the never found before all-optical "push-broom" CSs with the improved model.

The organization of the paper is as follows: Section II outlines the mathematical model of CS in VCSEL-SA-FSF and subsequently the stability analysis of CSs. The generation of CS molecules and the role of cavity parameters on them is studied extensively in Section III. A novel all-optical "push-broom effect" is demonstrated in Section IV, followed by a conclusion.

\section{Mathematical Model}

Our proposed model comprises of a VCSEL with SA, coupled with the FSF element provided by Bragg's reflector with negligible delay. Such optically injected broad area semiconductor cavity, exhibiting higher order saturation nonlinearity and coupled with FSF configuration can be represented by the following dimensionless dynamical equations:

$$
\dot{E}=\left[-(1-i \theta)+(1-i \alpha) d_{a}+(1-i \beta) d_{p}+i \Delta_{\perp}\right] E+F,
$$

$\dot{d_{a}}=c_{a}\left[d_{a}\left(1+|E|^{2}\right)-\mu\right]$,

$\dot{d}_{p}=c_{p}\left[d_{p}\left(1+s|E|^{2}\right)+\gamma\right]$,

$$
\dot{F}=-\left(\lambda+i \Omega_{0}\right) F+\sigma \lambda E \text {. }
$$

Here, $E(r, t)$ is the slowly varying amplitude of electric field and $F(r, t)$ is the feedback field. While $r$ (typically measured in $\mu \mathrm{m}$ ) is the spatial coordinate, $t$ (typically measured in $n s$ ) is the normalized time scaled to the round trip time of CS inside the cavity. The system parameters used in (1)-(4) are as follows: detuning parameter $\theta$ measures the coupling of optically injected field to the feedback field, $\mu$ and $\gamma$ are pump parameters for active and passive materials, respectively. $\alpha$ and $\beta$ are the line-width enhancement factors for active and passive materials, respectively, and usually posses large positive values for semiconductor devices, so far, VCSELs. On right hand side of (1), the first term describes the linear loss in the system, the second and the third terms represent the carrier densities in active and passive materials, respectively. The fourth term is due to the diffraction, with $\Delta_{\perp}=r^{(1-D)} \frac{\partial}{\partial r}\left(r^{(D-1)} \frac{\partial}{\partial r}\right)$ being the transverse component of diffraction operator. $d_{a}$ and $d_{p}$ represent the carrier densities in the active and passive medium, respectively. Parameters $c_{a}$ and $c_{p}$ are the ratio of the photon lifetime and carrier lifetime in active and passive materials, respectively. It is assumed that $c_{a}>c_{p}$, as the inverse of the carrier lifetime increases with the carrier density in the semiconductor material [12]. $s$ represents saturation strength of the nonlinearity and is defined as $s=\left(a_{p} c_{a}\right) /\left(a_{a} c_{p}\right)$, where, $a_{a}$ and $a_{p}$ represent the slopes of differential gain and are functions of carrier densities in active and passive materials, respectively [12]. $\sigma$ represents the strength of feedback field and assumes a value between 0 and 1. The frequency selection of feedback field is accomplished by a filter. $\lambda$ represents the band-width of filter reflection and $\Omega_{0}$ represents the corresponding resonant frequency [1], [12], [35]. A pump of suitable duration and transverse profile excites up the CS in the cavity, while the continuous feedback helps in sustaining such localized structure (CS). The trivial HSS solution for the system of equations (1)-(4) are as follows:

$$
E_{0}=0, d_{a 0}=\mu, d_{p 0}=-\gamma, F=0 .
$$

The non-trivial HSS solution for the system is determined as follows:

$$
\begin{aligned}
E_{0} & =\sqrt{I_{0}} e^{i\left[q x-\left(\omega+q^{2}\right) t\right]}, \\
d_{a 0} & =\mu /\left(1+I_{0}\right), \\
d_{p 0} & =-\gamma /\left(1+s I_{0}\right) . \\
F & =\sigma \lambda E /\left(\lambda+i \Omega_{0}\right) .
\end{aligned}
$$

Here, $q$ is the transverse wave vector and hence (6) represents a tilted traveling wave solution. Equation (6) can be further simplified to $E_{0}=\sqrt{I_{0}} e^{-i \omega t}$ by setting $q=0$. This is valid for a system having no wave-vector selection mechanism [12]. Also, the absence of diffusion term preserves the degeneracy of wave-vector. Considering the non-trivial HSS solution of carrier densities in active and passive materials in semiconductor cavity [(7) and (8) respectively], and feedback field (9), (1) turns to:

$$
\begin{aligned}
\frac{\partial E}{\partial t}= & {\left[-(1-i \theta)+\frac{\mu(1-i \alpha)}{1+|E|^{2}}-\frac{\gamma(1-i \beta)}{1+s|E|^{2}}+i \Delta_{\perp}\right] E } \\
& +(a-i b) E,
\end{aligned}
$$

with, $a=\sigma \lambda^{2} /\left(\lambda^{2}+\Omega_{0}^{2}\right)$ and $b=\sigma \lambda \Omega_{0} /\left(\lambda^{2}+\Omega_{0}^{2}\right)$.

Equation (10) resembles with CGLE and will be used henceforth. Equating the real parts on both sides of (10) leads to the 


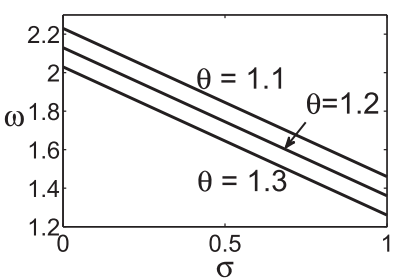

(a)

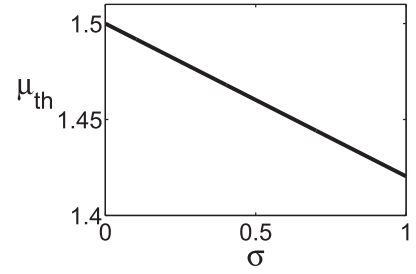

(b)
Fig. 1. (a) Variation of reference frequency $\omega$ with respect to feedback strength $\sigma$ at laser threshold with different detuning strength. (b) Variation of threshold pump power with respect to the feedback strength. Here $\alpha=2.7, \mu=1.37$, $\gamma=0.5, \beta=0, s=10, \lambda=0.5, \Omega_{0}=1.7$.

following relation of field intensity $I_{0}$ with the system parameters are obtained:

$$
\frac{\mu}{1+I_{0}}=1-a+\frac{\gamma}{1+s I_{0}},
$$

The imaginary part of (10) is too small to modify the diffraction coefficient, thus, can be neglected. However, the real part is crucial for the determination of the diffraction coefficient [12], [36]. The reference frequency $(\omega)$ of the system is controlled by the imaginary part of the governing equation (10) and is determined as follows:

$$
\omega=\alpha(1-a)-b-\theta+\frac{\gamma(\alpha-\beta)}{1+s I_{0}} .
$$

Like in VCSEL-SA system, in VCSEL-SA-FSF system too, the frequency is intensity dependent only for $\alpha \neq \beta$ (i.e. unequal line-width enhancement factor for active and passive materials). Besides, the $\omega$ is dependent on the feedback field parameters ( $\sigma$, $\Omega_{0}$ and $\lambda$ ) and detuning parameter $\theta$. Equation (12) clearly indicates that the inclusion of FSF decreases the reference frequency. Particularly, increase in feedback strength linearly decreases the value of $\omega$ with a slope of -0.770 [Fig. 1(a)]. For a given feedback strength, the reference frequency linearly decreases with increase in detuning. From (11), the laser threshold $\left(I_{0}=0\right)$ is found to be:

$$
\mu_{\mathrm{th}}=1-a+\gamma .
$$

Inclusion of FSF in the system reduces the threshold value of $\mu$ (i.e., $\mu_{t h}$ ). The reduction of $\mu_{t h}$ with respect to $\sigma$ is linear with a slope of -0.079 [Fig. 1(b)]. Substitution of laser threshold condition (13) to (11) provides the limit for the saturation parameter $s$ as:

$$
s>1+\frac{1-a}{\gamma} .
$$

The above is an essential condition for the bistability of the system. When plotted with respect to pump parameter $\mu$, the nontrivial HSS solution becomes parabolic in shape [Fig. 2(a)] and hence leads to the bistability. The turning point of the parabolic curve occurs at a pump value of:

$$
\mu_{t p}=\frac{(\sqrt{(1-a)(s-1)}+\sqrt{\gamma})^{2}}{s} .
$$

Thus $\mu_{t p}$ is controlled by parameters $s, \gamma, \sigma, \lambda$ and $\Omega_{0}$. Particularly, $\mu_{t p}$ linearly decreases with increase in $\sigma$ (Fig. 2(b)

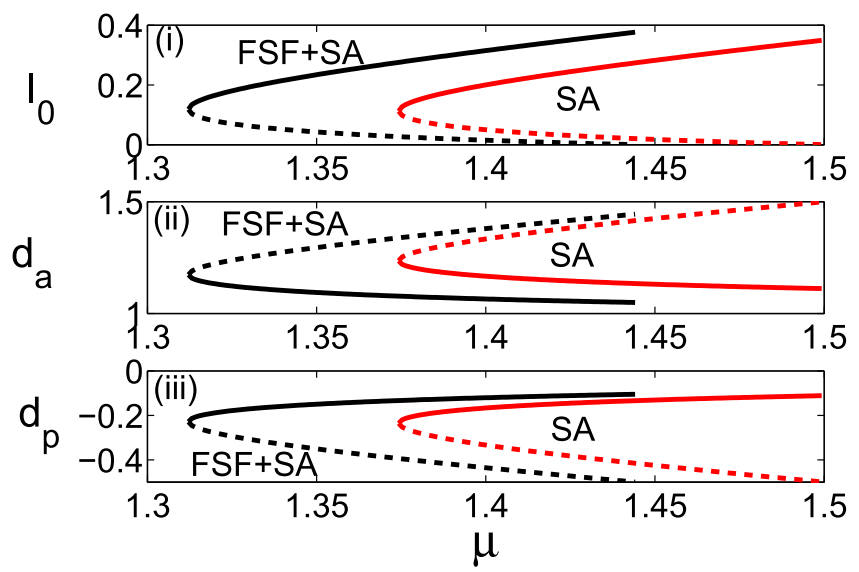

(a)

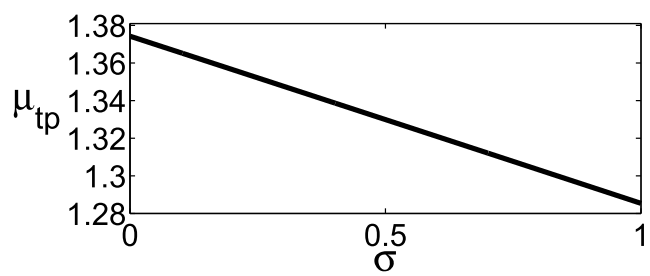

(b)

Fig. 2. HSSs solution with and without FSF in VCSEL-SA model: (a) $(i)$ the field intensity, ( ii) carrier density for active medium, (iii) carrier density for the passive medium, and (b) linear variation of turning point value of pump power with respect to the feedback strength at $\sigma=0.7$, rest of parameters are kept same as in Fig. 1.

with slope $=-0.088$ ). Fig. 2(a) provides the idea of stability as well as comparison between models with and without FSF. While, $\mu_{t h}=1.5$ and $\mu_{t p}=1.347$ for VCSEL-SA model, we determine $\mu_{t h}=1.458$ and $\mu_{t p}=1.327$ for VCSEL-SA-FSF model [Fig. 2(a)]. Therefore, inclusion of FSF lowers both the threshold and turning point values of pump power. Yet the range of stable CS, i.e., $\left(\Delta \mu=\mu_{t h}-\mu_{t p}\right)$, ultimately extends from 0.126 to 0.131 (i.e. nearly $4 \%$ increment). This is calculated for fixed feedback strength of $\sigma=0.7$. As large as $6 \%$ enhancement of $\Delta \mu$ is found by increasing the feedback strength. Similar extension is observed for both $d_{a}$ and $d_{p}$ [Figs. 2(a)(ii) and (iii)]. The positive slope branch (upper solution branch) of the curves of Fig. 2(a) is Hopf unstable if $\mu<\mu_{H}$, where $\mu_{H}=\gamma s$ and $\mu_{H}$ assumes same value as stated in [12]. The HSS is stable only if $\mu<\mu_{t h}$. The negative slope branch (lower solution branch) is always unstable. These three regions are identified in Fig. 3(a)(c). In Hopf instable region, the eigenvalues determined for the system are purely complex. The stable limit cycle decays to stable spiral or unstable spiral as the system moves away from stable region to the Hopf instable region. A comparison with similar figures for a VCSEL-SA [12] reveals that Hopf instability region increases with the inclusion of FSF in the system.

\section{Cavity Soliton Molecules}

With the knowledge of the stable parametric zone, we now explore the existence and dynamics of CS in the system. The governing equation (10) is solved numerically by the split-step 


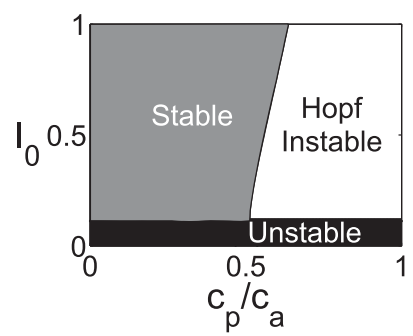

(a)

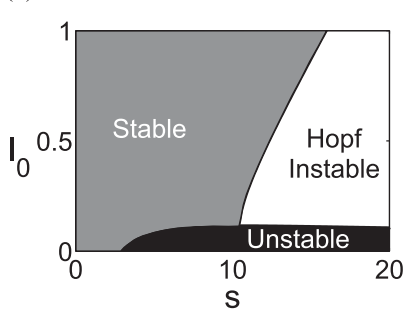

(c)

Fig. 3. Stability plots showing the stable, Hopf instable and unstable regions for VCSEL-SA-FSF. (a) $s=10, \gamma=0.5$, (b) $c_{p}=0.005, s=10$, (c) $c_{p}=$ $0.005, \gamma=0.5$, with $c_{a}=0.01, \sigma=0.7$ and rest of the parameters are as in Fig. 1 .

Fourier method (SSFM) with parameters chosen from the stable region. Throughout the numerical study, the step-size is fixed to 0.01 , and simulation is performed for 10 soliton periods. We consider the following unchirped "sech" function as the profile of CS:

$$
E(x, t)=A \operatorname{sech}(K r) e^{-i \omega t},
$$

where, $A$ represents the peak amplitude of CS and always assumes positive non-zero values, $K$ represents the inverse width and $\omega$ is the angular frequency (a real constant). The most important system parameters those significantly control CS dynamics are the feedback strength $\sigma$ and resonance frequency $\Omega_{0}$. As discussed in stability analysis, the saturation parameter $s$ has to follow the inequality condition (14), and $s$ assumes the value greater than unity. Line-width enhancement factor for active material should always be greater as compared to that of passive material $(\alpha>\beta)$. Considering this condition, the onset of Turing instability can be eluded [12]. Pump parameters for both active and passive medium $(\mu$ and $\gamma$ ) should follow the conditions stated in the stability analysis (13). In the current investigation, we set $\beta=0$. The values of the rest normalized parameters are set as $\alpha=2.7, \mu=1.37, \gamma=0.5, s=10$ and $\theta=1.3$, unless mentioned otherwise. Generally, a highly intense field undergoes initial perturbations and splits into almost equal amplitude branches, and then retain their shape, size and amplitude (Fig. 4). However, another perspective suggests that the velocity matching of the interacting CSs during interaction increases the chance of splitting the initial pulse into two or four CSs [37]. Also, the presence of higher order nonlinearities supports fission, fusion and annihilation of dissipative solitons [30]. Our numerical investigation (Fig. 4) gives rise to different stable bound state structures comprising of two or more CSs. For example, with $A=0.6278$ and $\sigma=0.7$, such bound state CSs are formed for $\Omega_{0}>1.97$ (Fig. 4). Precisely, two-CS

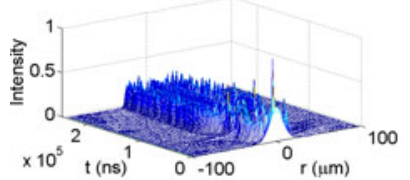

(a)

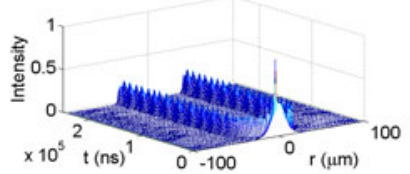

(c)

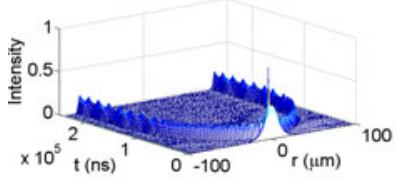

(e)

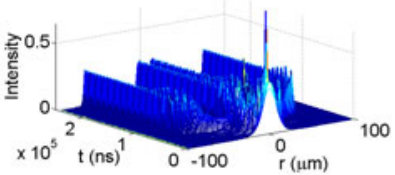

(b)

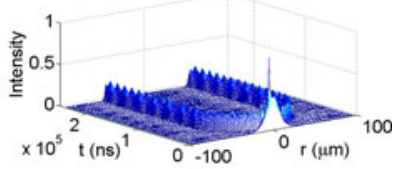

(d)

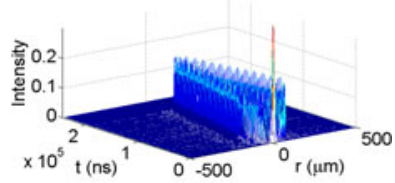

(f)
Fig. 4. Effect of variation of resonant frequency $\left(\Omega_{0}\right)$ (a) $\Omega_{0}=1.98$, (b) $\Omega_{0}=1.99$, (c) $\Omega_{0}=2.2$, (d) $\Omega_{0}=2.5$, (e) $\Omega_{0}=2.8$ and (f) $\Omega_{0}=3$. Here, $\sigma=0.7, A=0.6278$ and other parameters are same as in Fig. 1. Corresponding contour plots are shown in supplementary Fig. 1.

bound state is formed for $1.99<\Omega_{0}<3$ [Figs. 4(c)-(e)]. The CSs of the bound state vibrate periodically. As these CSs are born from same source, they can be considered as coherent pairs [38]. Such bound state of CSs can be referred as linear diatomic CS molecules. The oscillating CS atoms has potential application in tuning the optical clocks [13]. At a lower $\Omega_{0}$ (1.98 and 1.99), four-atom CS molecules are formed [Figs. 4(a) and (b)]. $\Omega_{0} \geqslant 3$ leads to the annihilation of CSs. The annihilation occurs due to the phase difference developed in the CSs. However, this annihilation can be opposed by increasing $\sigma$ (supplementary Fig. 2). As $\sigma$ increases the di-atomic CS molecule tends to get stabilize (supplementary Fig. 2).

The role of $\sigma$ can be well understood by keeping $\Omega_{0}$ constant. With increase in the $\sigma$, the distance between the CSs decreases. $\sigma$ can even control the number of atoms in the CS molecules. To show the effect with a bigger range of $\sigma$, we set a lower value of $A(=0.527)$. Diatomic CS molecules [Figs. 5(a)(d)] are found for $0.25<\sigma<0.35$. Higher value of $\sigma$ leads to the formation of linear tetra-atomic CS molecules [Figs. 5(e) and $(\mathrm{g})$ ]. Even triatomic linear CS molecules are possible for a particular $\sigma=0.49$ [Fig. 5(f)]. At very large $\sigma$ value, CS molecules get annihilated. Formation of CS molecules is observed with different values of $A$ as lower as 0.27 (supplementary Fig. 3). The limiting value of $\sigma$ for which CS molecules survives catastrophic collapse, however, is dependent on the peak amplitude $A$.

Till now, we obtain CSs of bell-shaped profile. Interesting $\mathrm{CS}$ profiles are found in the form of a $\cap$-shaped $[A=0.2667$, Fig. 6(a) $]$ and flat-top $[A=0.667$, Fig. 6(b) $]$ CS. Flat-top solutions for CGLE are generated from the general soliton solutions by transition of the roots of the general solution. As the two distinct positive soliton-solutions of a system approach each other, the soliton shape experiences a transition from standard shape (sech/bell-shape) to a flat-top shape. When two distinct real roots of the solution of CGLE become equal to each other, fronts are 


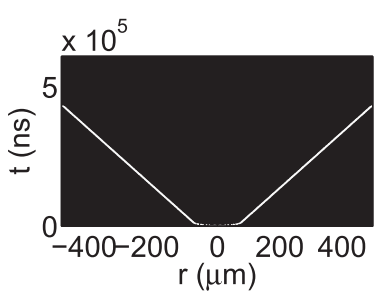

(a)

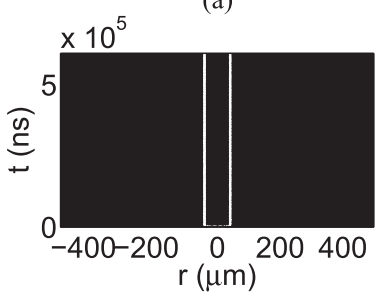

(c)

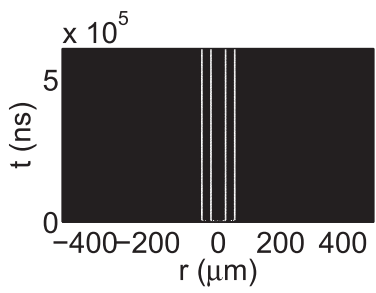

(e)

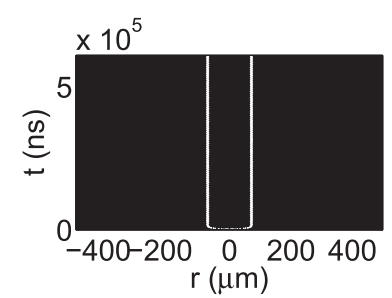

(b)

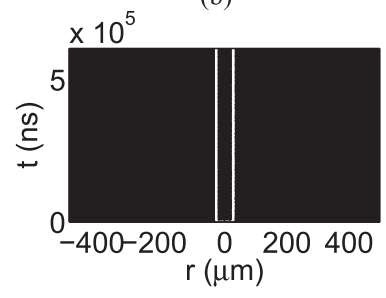

(d)

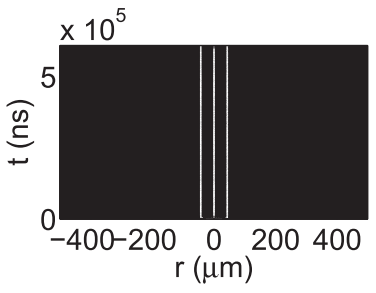

(f)

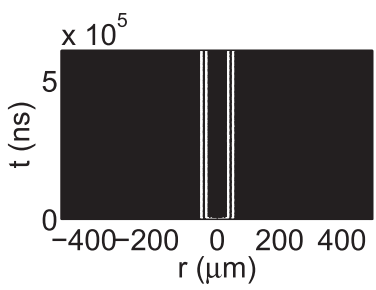

(g)

Fig. 5. The effect of feedback strength parameter $\sigma$ on CS at $\Omega_{0}=1.7$ and $A=0.527$. (a) $\sigma=0.25$, (b) $\sigma=0.26$, (c) $\sigma=0.31$, (d) $\sigma=0.33$, (e) $\sigma=0.46$, (f) $\sigma=0.48$ and (g) $\sigma=0.49$, keeping rest of the parameters same as in Fig. 1.

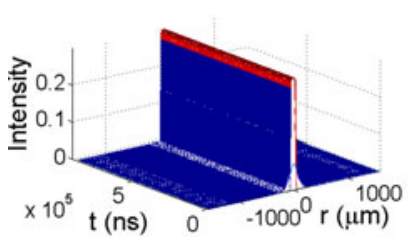

(a)

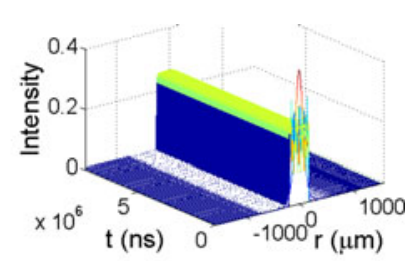

(b)
Fig. 6. (a) Generation of $\cap$-shaped CS with $A=0.667$. (b) Flat-top CS with $A=0.2667$. Here, $\sigma=0.7, \Omega_{0}=2.12, K=100$ and keeping rest of the parameters same as in Fig. 1.

formed. The pulse width becomes infinite, and it fragments into two fronts. The phase assumes constant value in the front region [39], [40]. Fig. 7 identifies $\left(A, \Omega_{0}\right)$ parametric region of different soliton-solutions, such as, flat-top, fronts, $\cap$-shaped and region in which the input field decays. Although, CGLE possesses different solutions in the form of fronts and pulses, change in parameter values can easily transform one solution into others [39]. In the present case, the transition of CS-solution

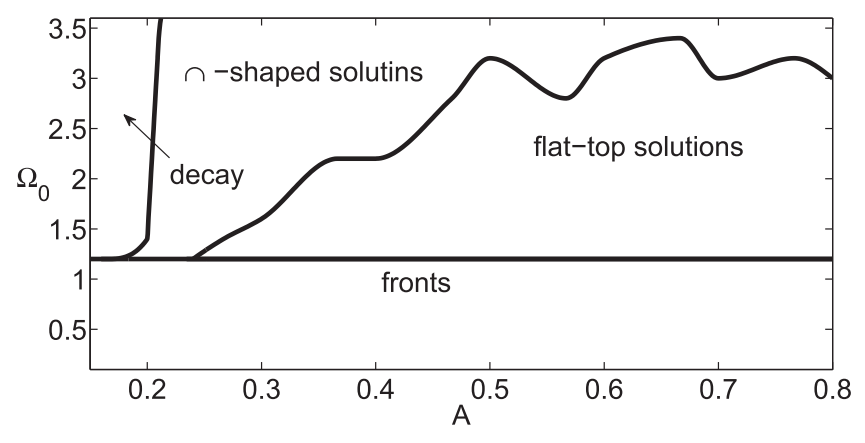

Fig. 7. Regions for $\cap$-shaped, fronts and flat-top solutions in $A-\Omega_{0}$ plane for $\sigma=0.7$. Here $\alpha=2.7, \mu=1.37, \gamma=0.5, \beta=0, s=10, \lambda=0.5$ and $K=100$.

shape is shown in terms of resonant frequency of feedback $\Omega_{0}$ and input field amplitude $A$ at a constant feedback strength $\sigma$ (Fig. 7). Here, $\sigma=0.7, \Omega_{0}=2.12, A=0.667$ and $K=100$. Fronts are formed for $\Omega_{0}<1$, flat-top solutions are obtained for $1.0<\Omega_{0}<3.0$. The amplitude and width of CS decreases with the increase in $\Omega_{0}$-value. For $A=0.2667$, fronts are formed for $\Omega_{0} \leq 1$, a flat-top is obtained for $1.0<\Omega_{0}<1.5$ and $\cap$-shaped CS for $1.5<\Omega_{0}<2.6$. For $\Omega_{0}>2.6$, the CS decays quickly. The parameter $\Omega_{0}$ has significant impact on the amplitude of the CS during evolution. As the value of $\Omega_{0}$ increases, the evolution amplitude increases, but on the other hand the soliton width decreases significantly.

The experimental realization of CS uses a VCSEL typically of diameter $200 \mu \mathrm{m}$ (while effective cavity length is $\approx$ few $\mu \mathrm{m}$ ) or a vertical cavity semiconductor amplifier of diameter $\approx 150 \mu \mathrm{m}$. Naturally, a larger diameter device is more suitable for observing transverse CS dynamics. A variety of experimental schemes to excite CS are available in [9], [17], [18], and [21]. Our theoretical results suggest a range of system parameters that includes the ones for which CSs have been achieved in [9], [17], and [18]. Typical VCSEL is a selectively oxidized multilayer system which can be grown n-GaAs substrate. A half-wavelength thick GaAs layer in conjugation with multiple (each of thickness $\approx 8 \mathrm{~nm}$ ) compressively strained $I n_{0.2} G a_{0.8} A s$ quantum wells (QW) comprises the active layer that may yield an emission of $\approx 980 \mathrm{~nm}$. The number of the QW may vary, provided suitable separation barriers $(\approx 10 \mathrm{~nm}$ thick) are maintained. Alternatively, an $A l_{0.2} G_{0.8} A s$ active layer with unstrained $G a A s$ QWs may cause an emission at $850 \mathrm{~nm}$. A comprehensive documentation on configuration as well as operating principles can be found in [41]. The frequency-selection is carried out by a Lorentzian filter. Usually, a semiconductor saturable absorber mirror (SESAM); a semiconductor Bragg reflector that provides the FSF (having single reflection peak at $\approx 981 \mathrm{~nm}$ ) is coupled with the VCSEL through a self-imaging external cavity. The reflectivity is made high $(0.98-0.99)$ and reflection band width is made of the order of fraction of one $\mathrm{nm}(\approx 0.2 \mathrm{~nm}$ FWHM). Besides SESAM, carbon nanotubes and Graphene can be used as saturable absorber. For optically pumped VCSEL the pump intensity is typically few tens of $\mathrm{kW} / \mathrm{cm}^{2}$ and pump diameter is few tens of $\mu \mathrm{m}$. The line-width enhancement factors $(\alpha, \beta)$, band-width of filter reflection $(\lambda)$, detuning parameter $(\theta)$ and 


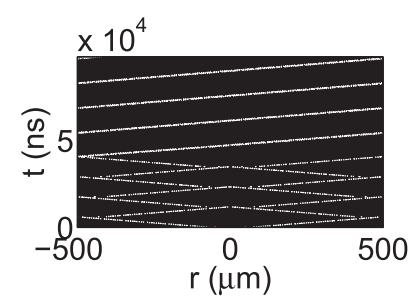

(a)

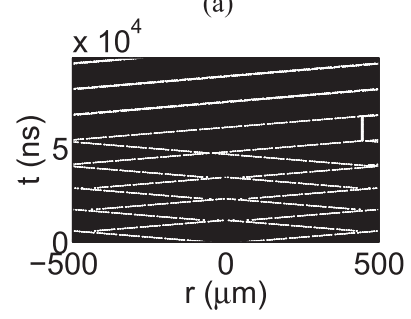

(c)

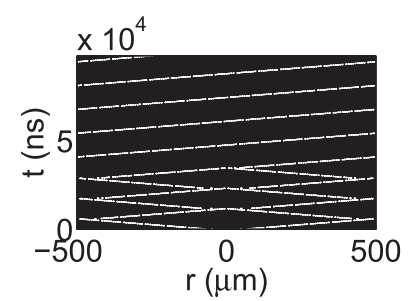

(b)

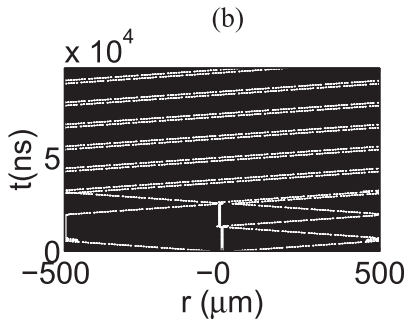

(d)

Fig. 8. All-optical push-broom: some selective push-broom dynamics of CS with increasing feedback strength. (a) $\sigma=0.61$, (b) $\sigma=0.64$, (c) $\sigma=0.65$ and (d) $\sigma=0.82$. For all panels $\beta=1, \Omega_{0}=2.12, A=0.2667$ and rest of the parameters are same as in Fig. 1. (Visuals enclosed).

feedback parameter $(\sigma)$ correspond physically at a time scale of $1 \mathrm{~ns}$. The typical size of the generated CS ranges $\approx 13 \mu \mathrm{m}$ in spatial scale. Since in the present investigation a primary CS experiences splitting into several CSs, the spot size of the resultant CS may be even smaller.

\section{Push-Broom EFFECT}

The previous section uses $\beta=0$ and although it is not a rule of thumb, the CSs are stationary in most of the cases. In contrary, for $\beta>0$, we witness intriguing dynamics of the generated CS. The most appealing one is presented in Fig. 8 where two CSs initially oscillate and eventually one of them starts pushing the other. Such phenomena is observed for a range of $\sigma$, i.e., $0.60<\sigma<0.83$, keeping $\beta=1$, $\Omega_{0}=2.12, A=0.2667$ and rest of the parameters same as in Section III. This, one of its kind, novel phenomenon can be termed as "push-broom" effect and is observed for first time. Notably the "push-broom" of [32]-[34] means heaping up of the energy of a weak probe by a strong pump pulse, not an actual manoeuvre of solitons. In the present case, one CS pushes the other and swipe along the transverse plane of the cavity, therefore, shows a "true" all-optical push-broom effect. The pushbroom effect is observed for very limited range of $\beta, \sigma$ and $\Omega_{0}$. With variation of $\sigma$, the dynamics also varies but eventually leads to push-broom. For $\sigma=0.61$ [Fig. 8(a)] and $\sigma=0.64$ [Fig. 8(b)] the initially colliding CSs never come to rest before showing push-broom. However, for $\sigma=0.65$ [Fig. 8(c)], $0.70,0.81$ and 0.82 [Fig. 8(d)] one of the colliding CS momentarily comes to rest. In the course of evolution when both the CSs collide and interact with each other, the phenomenon of cross-phase modulation (XPM) plays important role between the stationary and moving CS. Also, the asymmetric spectral broadening of interacting CSs is a resultant of the XPM. XPM generally occurs along with the self-phase modulation when

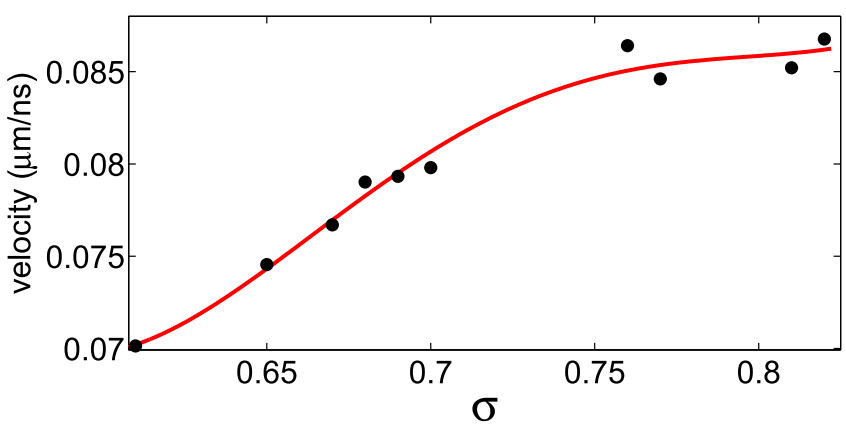

Fig. 9. Variation of the velocity of push-broom CS with the feedback strength. Points represent the numerical data and solid line represents the fitted data. Other parameters are same as in Fig. 8.

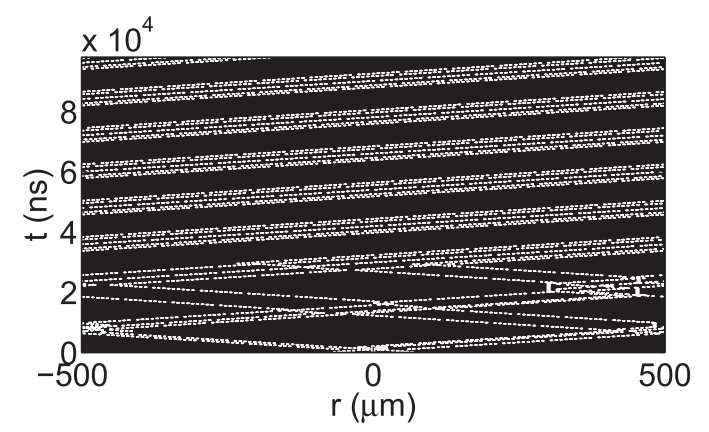

Fig. 10. Generation of "Marching CSs" for $\sigma=0.76$. Other parameters are same as in Fig. 8. (Visuals enclosed).

multiple localized structures simultaneously evolve in the cavity. The XPM induced coupling between interacting solitons results in modulation instability, thus, leads to the asymmetric spectral broadening. XPM may not be included explicitly in the initial incident field, but evolution dynamics of CSs encounter its effect [38]. As a result, the stationary CS is swept by the moving CS along with it. The velocity of the "push-broom" CSs significantly increases with feedback strength and eventually saturates (Fig. 9). Push-broom is also observed with more than two CSs. For example, at $\sigma=0.76$, the initial field splits into five CSs those eventually start moving in the same direction (Fig. 10), as if, they are marching. Such "marching" CSs maintain a constant gap during marching. The number of marching solitons can be controlled by the system parameters, like peak amplitude $A$ and resonant frequency $\Omega_{0}$. The marching speed can also be adjusted up to certain extent by tuning the feedback strength. By controlling the feedback strength, not only push-broom dynamics can be influenced but also a stationary CS molecule can be drifted along the transverse axis of the VCSEL. For an instance, a reduction in $\sigma$ from 0.84 to 0.83 leads to migration of a CS triatomic molecule by $150 \mu \mathrm{m}$ across the transverse plane of cavity (Fig. 11). Also, the symmetric triatomic CS molecules turns to asymmetric one. Few instances of CS migration may be short-lasting but a proper choice of parameters assures postmigration stability of CSs. The all-optical "push-broom" CSs, "marching" CSs and "migrating" CSs found in the present study are found to be controllable and predictable; thus can open a new horizons in all-optical devising. Since, any local defect, gradient or inhomogeneity of the material can be probed using such CSs, 


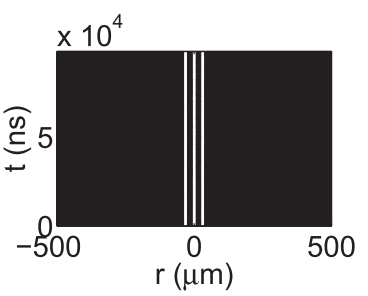

(a)

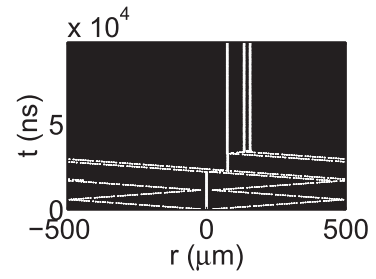

(b)
Fig. 11. (a) A stationary tri-atomic CS molecule for $\sigma=0.84$, (b) migration of the CS molecule for $\sigma=0.83$. Other parameters are same as in Fig. 8 . (Visuals enclosed).

they have potential for applications in soliton force microscopy, shift register and serial-parallel converter. Since it has multiple components a group of marching solitons, in principle, can be useful to accomplish quicker scanning of a spatially extended media. Migrated solitons can be used for memory relocation.

\section{Conclusion}

An improved system for hosting cavity soliton is modeled by coupling frequency-selective feedback to a VCSEL with saturable absorber. The inclusion of frequency-selective feedback lowers down the laser pump threshold and extends the operating range, thus making the system more energy efficient and easier for experimental verification. Parametric regimes for homogeneous stationary bistable CSs is identified analytically and verified numerically. Also regions of stable solitons, fronts and flat-top CSs are determined. A large family of linear di-atomic, tri-atomic and tetra-atomic CS molecule are found in absence of line-width enhancement for passive medium. A novel all-optical "push-broom" CSs, marching CSs and migrated CSs are found. While the parametric stability regions can be important for experimental realization of CS, the CS molecules may improve all-optical memory devices. The novel "push-broom" dynamics of CSs may find its applications in the soliton force microscope; a still emerging all-optical probing device.

\section{ACKNOWLEDGMENT}

The authors would also like to thank B. C. Mohanty for the support and encouragement. S. Jana would like to thank W. J. Firth and G.-L. Oppo for advices and several discussion sessions.

\section{REFERENCES}

[1] T. Ackemann, W. J. Firth, and G.-L. Oppo, "Fundamentals and applications of spatial dissipative solitons in photonic devices," in Advances in Atomic Molecular and Optical Physics, vol. 57, E. Arimondo, P. R. Berman, and C. C. Lin, Eds. New York, NY, USA: Academic, 2009, ch. 6, pp. 323-421.

[2] M. Eslami, R. Kheradmand, and F. Prati, "All-optical tunable delayline memory based on a semiconductor cavity-soliton laser," Phys. Rev. A, vol. 89, Jan. 2014, Art. no. 013818. [Online]. Available: http://link. aps.org/doi/10.1103/PhysRevA.89.013818

[3] X. Hachair et al., "Cavity solitons in broad-area vertical-cavity surface-emitting lasers below threshold," Phys. Rev. A, vol. 69, Apr. 2004, Art. no. 043817. [Online]. Available: http://link.aps.org/doi/ 10.1103/PhysRevA.69.043817
[4] M. Brambilla, L. A. Lugiato, F. Prati, L. Spinelli, and W. J. Firth, "Spatial soliton pixels in semiconductor devices," Phys. Rev. Lett., vol. 79, pp. 2042-2045, Sep. 1997. [Online]. Available: http://link.aps.org/ doi/10.1103/PhysRevLett.79.2042

[5] S. Barland et al., "Cavity solitons as pixels in semiconductor microcavities," Nature, vol. 419, pp. 699-702, Oct. 2002. [Online]. Available: http://dx.doi.org/10.1038/nature01049

[6] A. G. Vladimirov, A. Pimenov, S. V. Gurevich, K. Panajotov, E. Averlant, and M. Tlidi, "Cavity solitons in vertical-cavity surface-emitting lasers," Philosoph. Trans. Roy. Soc. London A, Math., Phys. Eng. Sci. vol. 372, no. 2027, 2014, Art. no. 20140013. [Online]. Available: http:// rsta.royalsocietypublishing.org/content/372/2027/20140013

[7] P. V. Paulau, A. J. Scroggie, A. Naumenko, T. Ackemann, N. A. Loiko, and W. J. Firth, "Localized traveling waves in vertical-cavity surfaceemitting lasers with frequency-selective optical feedback," Phys. Rev. E, vol. 75, May 2007, Art. no. 056208. [Online]. Available: http://link.aps. org/doi/10.1103/PhysRevE.75.056208

[8] A. J. Scroggie, W. J. Firth, and G.-L. Oppo, "Cavity-soliton laser with frequency-selective feedback," Phys. Rev. A, vol. 80, Jul. 2009, Art. no. 013829. [Online]. Available: http://link.aps.org/doi/10.1103/ PhysRevA.80.013829

[9] N. Radwell and T. Ackemann, "Characteristics of laser cavity solitons in a vertical-cavity surface-emitting laser with feedback from a volume bragg grating," IEEE J. Quant. Electron., vol. 45, no. 11, pp. 1388-1395, Nov. 2009.

[10] N. N. Rozanov, "Localized optical structures in the scheme of a nonlinear layer with a feedback mirror," Opt. Spectrosc., vol. 88, no. 2, pp. 238-241, 2000. [Online]. Available: http://dx.doi.org/10.1134/1.626785

[11] N. N. Rozanov, S. V. Fedorov, and A. N. Shatsev, "Pulsating solitons in a laser with relaxation of gain and saturable absorption," Opt. Spectrosc., vol. 91, no. 2, pp. 232-234, 2001. [Online]. Available: http:// dx.doi.org/10.1134/1.1397844

[12] M. Bache et al., "Cavity soliton laser based on VCSEL with saturable absorber," Appl. Phys. B, vol. 81, no. 7, pp. 913-920, 2005. [Online]. Available: http://dx.doi.org/10.1007/s00340-005-1997-9

[13] M. Eslami, R. Kheradmand, P. Bahari, and H. Tajalli, "Twin laser cavity solitons in a vesel with saturable absorber," Eur. Phys. J. D, vol. 69, no. 9, 2015, Art. no. 222. [Online]. Available: http://dx.doi.org/ 10.1140/epjd/e2015-60261-x

[14] A. Pimenov, A. G. Vladimirov, S. V. Gurevich, K. Panajotov, G. Huyet, and M. Tlidi, "Delayed feedback control of self-mobile cavity solitons," Phys. Rev. A, vol. 88, Nov 2013, Art. no. 053830. [Online]. Available: http://link.aps.org/doi/10.1103/PhysRevA.88.053830

[15] K. Panajotov and M. Tlidi, "Spontaneous motion of cavity solitons in vertical-cavity lasers subject to optical injection and to delayed feedback," Eur. Phys. J. D, vol. 59, no. 1, pp. 67-72, 2010. [Online]. Available: http://dx.doi.org/10.1140/epjd/e2010-00111-y

[16] M. Tlidi, A. G. Vladimirov, D. Pieroux, and D. Turaev, "Spontaneous motion of cavity solitons induced by a delayed feedback," Phys. Rev. Lett., vol. 103, Sep. 2009, Art. no. 103904. [Online]. Available: http://link.aps.org/doi/10.1103/PhysRevLett.103.103904

[17] T. Ackemann, N. Radwell, Y. Noblet, and R. Jäger, "Disorder mapping in VCSELs using frequency-selective feedback," Opt. Lett., vol. 37, no. 6, pp. 1079-1081, Mar. 2012. [Online]. Available: http://ol.osa. org/abstract.cfm?URI=ol-37-6-1079

[18] Y. Tanguy, T. Ackemann, W. J. Firth, and R. Jäger, "Realization of a semiconductor-based cavity soliton laser," Phys. Rev. Lett., vol. 100, Jan. 2008, Art. no. 013907. [Online]. Available: http://link.aps.org/ doi/10.1103/PhysRevLett.100.013907

[19] T. Maggipinto, M. Brambilla, G. K. Harkness, and W. J. Firth, "Cavity solitons in semiconductor microresonators: Existence, stability, and dynamical properties," Phys. Rev. E, vol. 62, pp. 87268739, Dec. 2000. [Online]. Available: http://link.aps.org/doi/10.1103/ PhysRevE.62.8726

[20] Y. Tanguy, N. Radwell, T. Ackemann, and R. Jäger, "Characteristics of cavity solitons and drifting excitations in broad-area vertical-cavity surface-emitting lasers with frequency-selective feedback," Phys. Rev. A, vol. 78, Aug. 2008, Art. no. 023810. [Online]. Available: http://link. aps.org/10.1103/PhysRevA.78.023810

[21] Y. Menesguen, S. Barbay, X. Hachair, L. Leroy, I. Sagnes, and R. Kuszelewicz, "Optical self-organization and cavity solitons in optically pumped semiconductor microresonators," Phys. Rev. A, vol. 74, Aug. 2006, Art. no. 023818. [Online]. Available: http://link.aps.org/ doi/10.1103/PhysRevA.74.023818 
[22] A. J. Scroggie, J. M. McSloy, and W. J. Firth, "Self-propelled cavity solitons in semiconductor microcavities," Phys. Rev. E, vol. 66, Sep. 2002, Art. no. 036607. [Online]. Available: http://link.aps.org/ doi/10.1103/PhysRevE.66.036607

[23] F. Pedaci, P. Genevet, S. Barland, M. Giudici, and J. R. Tredicce, "Positioning cavity solitons with a phase mask," Appl. Phys. Lett., vol. 89 , no. 22, pp. 1-3, 2006. [Online]. Available: http://scitation.aip. org/content/aip/journal/apl/89/22/10.1063/1.238886 7

[24] P. Parra-Rivas, D. Gomila, L. Gelens, M. A. Matias, and P. Colet, "Dynamics of dissipative solitons in presence of inhomogeneities and drift," in Nonlinear Optical Cavity Dynamics: From Microresonators to Fiber Lasers. Hoboken, NJ, USA: Wiley, 2016, ch. 5, pp. 107-128. [Online] Available: http://dx.doi.org/10.1002/9783527686476.ch5

[25] T. Ackemann et al., "Dynamics of dissipative solitons in presence of inhomogeneities and drift," in Nonlinear Optical Cavity Dynamics: From Microresonators to Fiber Lasers. Hoboken, NJ, USA: Wiley, 2016, ch. 3, pp. 41-76. [Online]. Available: http://dx.doi.org/ $10.1002 / 9783527686476 . c h 3$

[26] O. A. Egorov and F. Lederer, "Spin-induced motion of cavity polariton soliton molecules," in Proc. 2013 IEEE 2nd Int. Workshop Nonlinear Photon., 2013, pp. 34-35.

[27] C. Ma, X. Tian, B. Gao, and G. Wu, "Dynamic evolution of the soliton molecules in an all-normal dispersion fiber laser," Laser Phys., vol. 27, no. 6, 2017, Art. no. 065102. [Online]. Available: http://stacks. iop.org/1555-6611/27/i=6/a $=065102$

[28] Y. Luo et al., "Group-velocity-locked vector soliton molecules in fiber lasers," Sci. Rep., vol. 7, 2017, Art. no. 2369.

[29] N. N. Akhmediev, A. Ankiewicz, and J. M. Soto-Crespo, "Multisoliton solutions of the complex Ginzburg-Landau equation," Phys. Rev. Lett., vol. 79, pp. 4047-4051, Nov. 1997. [Online]. Available: http://link. aps.org/doi/10.1103/PhysRevLett.79.4047

[30] G. I. Stegeman and M. Segev, "Optical spatial solitons and their interactions: Universality and diversity," Science, vol. 286, no. 5444 pp. 1518-1523, 1999. [Online]. Available: http://science.sciencemag. org/content/286/5444/1518

[31] B. Luther-Davies, Spatial Solitons in Saturating Nonlinear Optical Materials. Dordrecht, The Netherlands: Springer, 2001, pp. 115-139.

[32] C. M. de Sterke, "Optical push broom," Opt. Lett., vol. 17, no. 13, pp. 914-916, Jul. 1992. [Online]. Available: http://ol.osa.org/abstract. cfm?URI $=$ ol-17-13-914
[33] M. J. Steel and C. Martijn de Sterke, "Schrödinger equation description for cross-phase modulation in grating structures," Phys. Rev. A, vol. 49, pp. 5048-5055, Jun. 1994. [Online]. Available: http://link. aps.org/doi/10.1103/PhysRevA.49.5048

[34] N. G. R. Broderick, D. Taverner, D. J. Richardson, M. Ibsen, and R. I. Laming, "Optical pulse compression in fiber Bragg gratings," Phys. Rev. Lett., vol. 79, pp. 4566-4569, Dec. 1997. [Online]. Available: http://link. aps.org/doi/10.1103/PhysRevLett.79.4566

[35] W. J. Firth and P. V. Paulau, "Soliton lasers stabilized by coupling to a resonant linear system," Eur. Phys. J. D, vol. 59, no. 1, pp. 13-21, 2010. [Online]. Available: http://dx.doi.org/10.1140/epjd/e2010-00116-6

[36] S. V. Fedorov, A. G. Vladimirov, G. V. Khodova, and N. N. Rosanov, "Effect of frequency detunings and finite relaxation rates on laser localized structures," Phys. Rev. E, vol. 61, pp. 5814-5824, May 2000. [Online]. Available: http://link.aps.org/doi/10.1103/PhysRevE.61.5814

[37] H. Leblond, The Davey-Stewartson Model in Quadratic Media: A Way to Control Pulses. Dordrecht, The Netherlands: Springer, 2001, pp. 215-218.

[38] G. P. Agarwal, Nonlinear Fiber Optics, (Optics and Photonics), 4th ed. San Diego, CA, USA: Academic, 2006. [Online]. Available: http:// www.sciencedirect.com/science/book/9780123695161

[39] N. Akhmediev and A. Ankiewicz, Solitons. New York, NY, USA: Springer, 1997.

[40] N. N. Akhmediev, V. V. Afanasjev, and J. M. Soto-Crespo, "Singularities and special soliton solutions of the cubic-quintic complex GinzburgLandau equation," Phys. Rev. E, vol. 53, pp. 1190-1201, Jan. 1996. [Online]. Available: http://link.aps.org/doi/10.1103/PhysRevE.53.1190

[41] R. Michalzik and K. J. Ebeling, "Operating principles of VCSELs," in Vertical-Cavity Surface-Emitting Laser Devices (Springer Series in Photonics), vol. 6, H. Li and K. Iga, Eds. Berlin, Germany: Springer-Verlag, 2003, pp. 1-45 